\title{
Effect of alcohol consumption on nonalcoholic fatty liver disease
}

\author{
Gong Weng, Winston Dunn \\ Department of Internal Medicine, The University of Kansas Medical Center, Kansas City, KS, USA \\ Contributions: (I) Conception and design: All authors; (II) Administrative support: All authors; (III) provision of study materials or patients: All \\ authors; (IV) Collection and assembly of data: All authors; (V) Data analysis and interpretation: All authors; (VI) Manuscript writing: All authors; (VII) \\ Final approval of manuscript: All authors. \\ Correspondence to: Gong Weng. The University of Kansas Medical Center, 3901 Rainbow Blvd, MS 2027, Kansas City, KS 66160, USA. \\ Email: gweng@kumc.edu.
}

\begin{abstract}
The prevalence of nonalcoholic fatty liver disease (NAFLD) is estimated to be $26.3 \%$ among the US population. A subset of this population exhibits an aggressive histological phenotype, nonalcoholic steatohepatitis (NASH) with $\geq$ stage 2 fibrosis, which may progress to cirrhosis. The definition of NAFLD excludes excessive alcohol intake, which is well known to cause alcoholic liver disease and will not be discussed here. Most NAFLD clinical trials use $~ 14$ drinks per week as the cutoff for excessive alcohol intake. Alcohol consumption below this threshold, which we define as moderate alcohol consumption, is common in the US. According to the 2012 Behavioral Risk Factor Surveillance System (BRFSS), 56\% of the US adult population consume alcohol, but only $8.2 \%$ report drinking heavily and $18.3 \%$ report binge drinking. The American Association for the Study of Liver Diseases (AASLD) Practice Guidance of 2018 states that there are insufficient data to make a recommendation with regard to moderate alcohol consumption in patients with NAFLD, citing a lack of longitudinal studies that examine the impact of moderate alcohol consumption on disease progression and its extrahepatic harms versus benefits, specifically in individuals with established NAFLD. NAFLD prevalence studies have generally noted a negative correlation between modest alcohol consumption and NAFLD. However, prevalence studies have limited application to patients with established NAFLD who present to the clinic. There can also be many confounding factors, because modest alcohol consumption is also negatively associated with some NAFLD risk factors, and those risk factors may not be adequately adjusted for in analyses. The prevalence of NASH with significant fibrosis ( $\geq$ F2) is more important because this is the group that is believed to have progressive disease. Thus, cohort studies of disease progression are more important from the patient's standpoint. Because these patients have already developed NAFLD or NASH, their interest lies in their odds of disease progression if they have moderate alcohol consumption compared to abstinence. It is also noteworthy that cardiovascular disease is the most important cause of death among patients with NAFLD. Moderate alcohol consumption has been associated with a reduction in overall mortality, but mostly in cardiovascular mortality. However, this protective effect has not been demonstrated specifically in patients with NAFLD.
\end{abstract}

Keywords: Nonalcoholic fatty liver disease (NAFLD); nonalcoholic steatohepatitis (NASH); alcohol

Received: 12 June 2019; Accepted: 27 August 2019; Published: 17 September 2019.

doi: $10.21037 /$ tgh.2019.09.02

View this article at: http://dx.doi.org/10.21037/tgh.2019.09.02 


\section{Alcohol and the prevalence of nonalcoholic fatty liver disease (NAFLD)}

In the general population, data from cross-sectional studies suggest that modest to moderate alcohol consumption is associated with a lower prevalence of NAFLD compared to abstinence from alcohol. Although these studies can be useful for guiding public policies, they have limited application to the patient with NAFLD or nonalcoholic steatohepatitis (NASH) who presents to the clinic. In a meta-analysis using data from eight different studies and their own Argentinean cohort, Sookoian et al. sought to delineate the association between alcohol intake and the prevalence of NAFLD (1). This study included 43,175 people who were divided into two groups, namely nondrinkers (defined as $0 \mathrm{~g}$ /day of alcohol intake; $\mathrm{n}=30,791$ ) and modest to moderate drinkers (defined as $<40 \mathrm{~g} /$ day of alcohol intake; $n=12,384$ ). The analysis showed that the group with modest to moderate alcohol intake had decreased odds of NAFLD compared to the non-drinkers. The authors concluded that modest to moderate alcohol intake is associated with a $31 \%$ protective effect against developing NAFLD. However, in a later meta-regression analysis incorporating data from six combined studies $(n=42,059)$, BMI was shown to be a potential confounder, because those who consumed a modest amount of alcohol appeared to have lower BMIs compared to abstainers (2). Nonetheless, the results suggest that modest alcohol consumption is inversely associated with the diagnosis of NAFLD for those without a known diagnosis of NAFLD.

\section{Alcohol and the prevalence of NASH}

$\mathrm{NASH}$ is a histologically advanced stage of NAFLD characterized by steatosis with inflammation and ballooning degeneration. Given that moderate alcohol consumption may protect against the development of NAFLD in the general population, this prompts the question of whether moderate alcohol consumption protects against NASH in those with established NAFLD.

In biopsy-proven NAFLD, moderate alcohol consumption appears to be associated with a lower prevalence of NASH and fibrosis when compared to abstinence in three independent study groups. A metaanalysis of 822 participants (550 drinkers and 272 nondrinkers) performed by Sookoian et al. (1), which incorporated data from the NASH Clinical Research Network (3) and their Argentinean cohort, showed a decreased prevalence of steatohepatitis and fibrosis in those with NAFLD among moderate drinkers compared to nondrinkers (OR: 0.501, 95\% CI: 0.340-0.740, $\mathrm{P}<0.0005$ ).

A study by Mitchell et al. of 187 patients with biopsyconfirmed NAFLD yielded similar results (4): modest consumption, which was defined in this study as $<70 \mathrm{~g} /$ week, was associated with lower fibrosis scores compared to abstinence (OR: 0.33, 95\% CI: 0.14-0.78, P=0.01) with a trend toward non-significance in the moderate consumption group, which was defined in this study as $\geq 70 \mathrm{~g} /$ week (OR: 0.16, 95\% CI: $0.02-1.11, \mathrm{P}=0.06$ ). This protective effect was not demonstrated in binge drinkers who consumed similar amounts of alcohol with respect to their non-binge drinking counterparts compared to abstainers.

Gene expression analysis from liver biopsies has revealed a marked inhibition of immune response pathways in modest drinkers compared to non-drinkers, which may account for the lowering of liver fibrosis and hepatocellular injury (5). A microarray hierarchical cluster analysis of biopsied liver tissue from Japanese patients showed a between-group difference in gene expression clusters, namely that the modest alcohol consumption group ( $<20 \mathrm{~g} /$ day) exhibited reduced expression of genes associated with several immune response pathways, most notably those involving monocytes. Subsequent RT-PCR revealed decreased expression of ICAM1 and PSMB9 in the modest alcohol consumption group and a trend toward lower TLR4 expression. Recent evidence has shown that a chronic inflammatory state induced by endogenous TLR ligands and their recruitment of macrophages is associated with increased adiposity $(6,7)$. Therefore, together these findings suggest that modest alcohol consumption may play a role in the suppression of the pro-inflammatory state in metabolic syndrome and in the lower prevalence of NASH.

There are several limitations to the studies that have reported a decreased prevalence of NASH among modest drinkers. Upon review, certain confounders have not been adequately adjusted for at the individual level in the above studies (e.g., BMI, insulin resistance, socioeconomic status, sex, cumulative exposure, reverse causation) (8). Drinking pattern also appears to be an important factor to consider, as binge drinking does not appear to have protective effects (4). In addition, some studies provide evidence that the type of alcoholic beverage may influence prevalence $(4,9)$, which raises the question of whether the protective effect is due to ethanol itself or other components of alcoholic beverages; it is unclear whether the above studies have adjusted for this possibility. Furthermore, the above studies are cross- 
sectional and therefore cannot draw a causal link between exposure and disease; longitudinal studies are needed to verify such a relationship.

\section{The effect of alcohol on disease progression}

One of the most important clinical questions regarding the effect of alcohol consumption on NAFLD is its effect on disease progression. The AASLD Practice Guidance published in 2018 notes that the natural history of NAFLD and ongoing alcohol consumption is largely unknown, citing a lack of longitudinal studies (10). Since its publication, there have been a few important longitudinal studies published that dispute the protective effect of alcohol on NAFLD progression. For example, Chang et al. (11) followed a large cohort of 57,254 Korean participants with ultrasound-confirmed NAFLD to determine the effect of alcohol on noninvasive NAFLD fibrosis markers FIB4, NFS, and APRI. Chang et al. reported that after adjusting for several confounding variables, including hsCRP, HOMA-IR, BMI, smoking status, regular exercise and educational level, the hazard ratios (HRs) for disease progression between light drinkers (1-9.9 g/day) and abstainers were 1.06, 1.09, and 0.99 for FIB4, NFS, and APRI, respectively. Similarly, the HRs for disease progression between moderate drinkers (10-29.9 g/day for men and 10-19.9 g/day for women) and abstainers were 1.29, 1.31, and 1.09 for FIB4, NFS, and APRI, respectively. These results suggest that even light alcohol consumption is associated with a small but statistically significant risk for disease progression, and that moderate alcohol consumption is associated with a moderate risk for disease progression in patients with NAFLD. A major concern with this study, however, is whether these noninvasive indices are valid markers of NAFLD progression.

The finding in the NASH CRN cohort that modest alcohol consumption is associated with a lower prevalence of NASH and less fibrosis (3) is only half of the story. A subsequent cohort study of the same patient population showed that modest alcohol consumption is associated with less NASH resolution but a similar improvement in fibrosis as abstinence (12), highlighting the fact that a crosssectional study and a cohort study can yield conflicting results in the same patient population.

To evaluate the cumulative lifetime effect of moderate alcohol consumption on histological outcomes, and to address the existing gap in the literature of a randomized control trial in modest alcohol consumption, Sookoian et al. (13) conducted a Mendelian randomization study using a genetic variant (rs1229984 A;G) in the alcohol dehydrogenase (ADH1B) gene, in which A allele carriers reported an average of $6 \mathrm{~g} /$ day less alcohol consumption. The $\mathrm{ADH} 1 \mathrm{~B}$ gene was not directly linked to the pathophysiology of NASH, and individuals reporting excessive alcohol intake (>30 g/day for men, >20 g/day for women) were excluded from the study. Therefore, the ADH1B gene served as a valid instrument for instrumental analysis of moderate alcohol consumption. This study found that moderate alcohol consumption was associated with a higher NAFLD-Activity Score among 466 patients with biopsy-confirmed NAFLD, suggesting that modest alcohol consumption might lead to disease progression in patients with established NAFLD.

In contrast, studies from Japanese employees undergoing health or insurance examinations have generally shown an overwhelming protective effect of alcohol consumption. For instance, Moriya et al. (14) measured disease progression via ultrasound in a cohort of 5,297 Japanese employees, of which $26.7 \%$ exhibited NAFLD at baseline. In this study group, alcohol consumption, even up to excessive amounts (>280 g/week in men), appeared to protect against the development of NAFLD. It is reasonable to speculate that the unique circumstance of employee health or insurance examination poses a selection bias, as this type of result is generally not replicated in neighboring countries.

\section{Alcohol and cardiovascular mortality}

Some patients with cardiovascular risk factors, such as NASH, may ask whether they can drink a modest amount of alcohol, because modest alcohol intake has been associated with lower overall mortality compared to abstinence or heavy drinking. A 2006 meta-analysis of 34 studies across more than 1 million subjects confirmed this mortality benefit (15). Of note, mortality risk was lowest at the low dose of 6-7 g/day for men and $4 \mathrm{~g} /$ day for women. Table 1 lists four of the largest cohort studies that have examined the effect of modest alcohol consumption on overall mortality. These studies show that the optimal dose of alcohol is at or below 1 standard drink per day. Increased cirrhosis mortality, along with cancers, suicides, and accidents, were noted when consumption increased to 2-3 drinks per day. A subgroup analysis in the largest cohort, the Cancer Prevention Study II, further showed that the mortality benefit of modest alcohol consumption may be limited to those who are $\geq 60$ years old and have a history of heart disease, hypertension, stroke, or diabetes at 
Table 1 Benefits and risks associated with alcohol consumption

\begin{tabular}{|c|c|c|c|c|}
\hline Cohort & $\mathrm{N}$ & Alcohol dose & $\begin{array}{l}\text { Subgroup that has } \\
\text { mortality benefit }\end{array}$ & $\begin{array}{l}\text { Amount of alcohol consumption } \\
\text { associated with increased mortality }\end{array}$ \\
\hline $\begin{array}{l}\text { American Cancer } \\
\text { Society Prospective } \\
\text { Study (16) }\end{array}$ & 276,802 & $\begin{array}{l}\leq 2 \text { drinks/day has a mortality benefit; } \\
1 \text { drink/day has optimum benefit }\end{array}$ & Age 40-59 & $\begin{array}{l}2 \text { drinks/day: cirrhosis, suicide; } \\
3 \text { drinks/day: all cancers, oral cavity } \\
\text { cancer, esophagus cancer, accidents }\end{array}$ \\
\hline $\begin{array}{l}\text { Cancer Prevention } \\
\text { Study II (17) }\end{array}$ & 490,000 & $\begin{array}{l}\leq 1 \text { drink/day has a mortality benefit; } \\
<1 \text { drink/day has optimum benefit }\end{array}$ & $\begin{array}{l}\text { Age } \geq 60 \text { with heart } \\
\text { disease, hypertension, } \\
\text { stroke, or diabetes at } \\
\text { baseline }\end{array}$ & $\begin{array}{l}\text { 2-3 drinks/day: cirrhosis, alcoholism, or } \\
\text { both; alcohol-related cancers }\end{array}$ \\
\hline $\begin{array}{l}\text { Nurses' Health } \\
\text { Study (18) }\end{array}$ & 121,700 & $\begin{array}{l}5.0-14.9 \mathrm{~g} / \text { day has a mortality } \\
\text { benefit; } 1.5-4.9 \mathrm{~g} / \text { day is the optimum } \\
\text { dose }\end{array}$ & Age $30-55$ & $\begin{array}{l}\geq 30 \mathrm{~g} / \text { day: all-cause mortality, cancer, } \\
\text { breast cancer, injury, cirrhosis }\end{array}$ \\
\hline
\end{tabular}

baseline (17). Furthermore, another large meta-analysis has shown that even moderate drinking is associated with certain types of cancers, including oropharyngeal cancer, esophageal squamous cell cancer, and female breast cancer (20).

\section{Discussion}

Although there is evidence to suggest that alcohol consumption protects against NAFLD development in the general population, findings on the effects of alcohol consumption among patients with NAFLD convey a more inconvenient reality. Most analyses on large cohorts of participants with established NAFLD indicate that alcohol consumption, even in modest amounts, leads to more disease progression, less disease improvement, and less $\mathrm{NASH}$ resolution. Beyond alcohol's role in liver disease, the evidence suggests that moderate alcohol consumption is associated with lower all-cause mortality. Cardiovascular mortality is more difficult to reconcile, especially given that cardiovascular disease is the most common cause of death among patients with NAFLD $(21,22)$. Given that these two disease processes share similar metabolic features, the methods to mitigate risk are also expected to be similar. For example, interventions such as diet, exercise, and weight loss benefit both cardiovascular and hepatic health. Given available evidence, and current lack thereof, regarding alcohol consumption in NAFLD, it would be difficult to recommend alcohol consumption as an approach to curtail risk, especially when certain other risk modifiers, such as diet, weight loss, and exercise, are universally touted as health promoting.

Beyond studies boasting its beneficial effects, alcohol has several detrimental effects. For example, alcohol consumption is associated with certain types of cancers (e.g., oral, esophageal, breast). In addition, both personal safety and development of dependence are risks of alcohol consumption. These risks have been noted above and in Table 1. Lest we be caught up in favoring one disease process over another, the existing evidence should caution clinicians against advocating for the use of alcohol in health promotion when it is non-essential to health.

\section{Acknowledgments}

None.

\section{Footnote}

Conflicts of Interest: The authors have no conflicts of interest to declare.

Ethical Statement: The authors are accountable for all aspects of the work in ensuring that questions related to the accuracy or integrity of any part of the work are appropriately investigated and resolved.

\section{References}

1. Sookoian S, Castano GO, Pirola CJ. Modest alcohol 
consumption decreases the risk of non-alcoholic fatty liver disease: a meta-analysis of 43175 individuals. Gut 2014;63:530-2.

2. Sookoian S, Pirola CJ. How Safe Is Moderate Alcohol Consumption in Overweight and Obese Individuals? Gastroenterology 2016;150:1698-703.e2.

3. Dunn W, Sanyal AJ, Brunt EM, et al. Modest alcohol consumption is associated with decreased prevalence of steatohepatitis in patients with non-alcoholic fatty liver disease (NAFLD). J Hepatol 2012;57:384-91.

4. Mitchell T, Jeffrey GP, de Boer B, et al. Type and Pattern of Alcohol Consumption is Associated With Liver Fibrosis in Patients With Non-alcoholic Fatty Liver Disease. Am J Gastroenterol 2018;113:1484-93.

5. Yamada K, Mizukoshi E, Seike T, et al. Light alcohol consumption has the potential to suppress hepatocellular injury and liver fibrosis in non-alcoholic fatty liver disease. PLoS One 2018;13:e0191026.

6. Jin C, Henao-Mejia J, Flavell RA. Innate immune receptors: key regulators of metabolic disease progression. Cell Metab 2013;17:873-82.

7. Hotamisligil GS. Inflammation and metabolic disorders. Nature 2006;444:860-7.

8. Ajmera VH, Terrault NA, Harrison SA. Is moderate alcohol use in nonalcoholic fatty liver disease good or bad? A critical review. Hepatology 2017;65:2090-9.

9. Dunn $W, \mathrm{Xu}$ R, Schwimmer JB. Modest wine drinking and decreased prevalence of suspected nonalcoholic fatty liver disease. Hepatology 2008;47:1947-54.

10. Chalasani N, Younossi Z, Lavine JE, et al. The diagnosis and management of nonalcoholic fatty liver disease: Practice guidance from the American Association for the Study of Liver Diseases. Hepatology 2018;67:328-57.

11. Chang Y, Cho YK, Kim Y, et al. Nonheavy Drinking and Worsening of Noninvasive Fibrosis Markers in Nonalcoholic Fatty Liver Disease: A Cohort Study. Hepatology 2019;69:64-75.

12. Ajmera V, Belt P, Wilson LA, et al. Among Patients With Nonalcoholic Fatty Liver Disease, Modest Alcohol Use

doi: $10.21037 / \operatorname{tgh} .2019 .09 .02$

Cite this article as: Weng G, Dunn W. Effect of alcohol consumption on nonalcoholic fatty liver disease. Transl Gastroenterol Hepatol 2019;4:70.
Is Associated With Less Improvement in Histologic Steatosis and Steatohepatitis. Clin Gastroenterol Hepatol 2018;16:1511-20.e5.

13. Sookoian S, Flichman D, Castano GO, et al. Mendelian randomisation suggests no beneficial effect of moderate alcohol consumption on the severity of nonalcoholic fatty liver disease. Aliment Pharmacol Ther 2016;44:1224-34.

14. Moriya A, Iwasaki Y, Ohguchi S, et al. Roles of alcohol consumption in fatty liver: a longitudinal study. J Hepatol 2015;62:921-7.

15. Di Castelnuovo A, Costanzo S, Bagnardi V, et al. Alcohol dosing and total mortality in men and women: an updated meta-analysis of 34 prospective studies. Arch Intern Med 2006; 166:2437-45.

16. Boffetta P, Garfinkel L. Alcohol drinking and mortality among men enrolled in an American Cancer Society prospective study. Epidemiology 1990;1:342-8.

17. Thun MJ, Peto R, Lopez A, et al. Alcohol consumption and mortality among middle-aged and elderly U.S. adults. N Engl J Med 1997;337:1705-14.

18. Fuchs CS, Stampfer MJ, Colditz GA, et al. Alcohol consumption and mortality among women. N Engl J Med 1995;332:1245-50.

19. Xi B, Veeranki SP, Zhao M, et al. Relationship of Alcohol Consumption to All-Cause, Cardiovascular, and CancerRelated Mortality in U.S. Adults. J Am Coll Cardiol 2017;70:913-22.

20. Bagnardi V, Rota M, Botteri E, et al. Light alcohol drinking and cancer: a meta-analysis. Ann Oncol 2013;24:301-8.

21. Angulo P, Kleiner DE, Dam-Larsen S, et al. Liver Fibrosis, but No Other Histologic Features, Is Associated With Longterm Outcomes of Patients With Nonalcoholic Fatty Liver Disease. Gastroenterology 2015;149:389-97.e10.

22. Ekstedt M, Hagstrom H, Nasr P, et al. Fibrosis stage is the strongest predictor for disease-specific mortality in NAFLD after up to 33 years of follow-up. Hepatology 2015;61:1547-54. 